\title{
Implantação do Programa de Assistência às Pessoas com Hipertensão Arterial Sistêmica na Estratégia Saúde da Familia'
}

\section{Implementation of the Program of Assistance to the Person with Hypertension in the Family Health Strategy}

Regina Lúcia Dalla Torre Silva' (D); Mayckel da Silva Barreto' (D); Guilherme Oliveira de Arruda² (D); Sonia Silva Marcon' (1)

'Universidade Estadual de Maringá (UEM) - Maringá (PR), Brasil.

${ }^{2}$ Universidade Federal do Mato Grosso do Sul (UFMS) - Coxim (MS), Brasil.
Como citar: Silva RLDT, Barreto MS, Arruda GO, Marcon SS. Implantação do Programa de Assistência às Pessoas com Hipertensão Arterial Sistêmica na Estratégia Saúde da Família. Cad Saúde Colet, 2021;29(3):366-375. https://doi. org/10.1590/1414-462X202129030002

\section{Resumo}

Introdução: Desde os anos 2000, existe no Brasil um plano para reorganização da atenção à hipertensão arterial, contudo ainda são escassos estudos que identifiquem e classifiquem seu grau de implantação. Objetivo: Avaliar o grau de implantação do Programa de Assistência às Pessoas com Hipertensão Arterial Sistêmica. Método: Pesquisa avaliativa realizada na Estratégia Saúde da Família do município de Maringá, Paraná, entre abril e junho de 2013, com os enfermeiros de 63 equipes. O instrumento utilizado avaliou as dimensões estrutural, prática assistencial e organização da atenção, com escore máximo de 337 pontos. Foram adotados tercis como critério para a classificação: incipiente ( $1{ }^{\circ}$ tercil), intermediário ( $2^{\circ}$ tercil) e avançado ( $3^{\circ}$ tercil). Resultados: Os escores obtidos variaram de 146,3 a 255,4 pontos. O Programa foi classificado como avançado em quatro equipes (6,3\%) e intermediário nas demais (93,7\%). Na dimensão estrutural, todas as equipes foram classificadas como avançadas; na prática assistencial, 16 equipes foram incipientes, 43, intermediárias, e 4, avançadas; na organização da atenção, 4 equipes foram incipientes, 58, intermediárias, e 1, avançada. Conclusão: Existem fragilidades na implantação do Programa em relação à organização da atenção e na assistência prestada aos pacientes, requerendo preparo profissional e atuação baseada em políticas públicas e protocolos assistenciais.

Palavras-chave: avaliação em saúde; hipertensão; atenção primária à saúde; desenvolvimento de programas; políticas públicas de saúde.

\begin{abstract}
Background: Since the 2000s, there has been a plan in Brazil for the reorganization of care for Arterial Hypertension. However, there are still few studies that identify and classify its degree of implementation. Objective: To assess the degree of implementation of the Assistance Program for People with Hypertension. Method:This is evaluative research carried out in the Family Health Strategy in the city of Maringá-Paraná, between April and June 2013, with nurses from 63 teams. The instrument used evaluated the structural dimensions, care practice, and organization of care, with a maximum score of 337 points. Tertiles were adopted as criteria for classification: incipient (1st tertile), intermediate (2nd tertile), and advanced (3rd tertile). Results: The scores obtained ranged from 146.3 to 255.4 points. The Program was
\end{abstract}

Trabalho realizado na Universidade Estadual de Maringá (UEM) - Maringá (PR), Brasil.

Correspondência: Sonia Silva Marcon. E-mail: soniasilva.marcon@gmail.com

Fonte de financiamento: Conselho Nacional de Desenvolvimento Científico e Tecnológico (CNPQ) - Edital Universal -

Processo 485807/2011-4.

Conflito de interesses: nada a declarar.

Recebido em: Jan. 06, 2019. Aprovado em: Jul. 20, 2020

'Trabalho originário da tese de doutorado intitulada "Avaliação da implantação da assistência às pessoas com hipertensão arterial em município do estado do Paraná - 2013", no ano de 2013, na Universidade Estadual de Maringá.

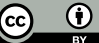

Este é um artigo publicado em acesso aberto (Open Access) sob a licença Creative Commons Attribution, que permite uso, distribuição e reprodução em qualquer meio, sem restrições desde que o trabalho original seja corretamente citado. 
classified as advanced in four teams (6.3\%) and intermediate in others (93.7\%). In the structural dimension, all teams were advanced; in care practice, 16 teams were incipient, 43 intermediate, and four advanced; in the organization of care, four teams were incipient, 58 intermediate, and one advanced. Conclusion: There are weaknesses in the implementation of the Program concerning the organization and care provided to patients, requiring professional training and action based on public policies and protocols.

Keywords: health evaluation; hypertension; primary health care; program development; health public policy.

\section{INTRODUÇÃO}

A hipertensão arterial sistêmica (HAS) é altamente prevalente em todo o mundo, o que acarreta grande demanda de assistência e eleva os custos médico-sociais'. No Brasil, em 2013, conforme aponta a Pesquisa Nacional de Saúde realizada por inquérito domiciliar, a prevalência de HAS autorreferida era de 21,4\%². Já segundo a Vigilância de Fatores de Risco e Proteção para Doenças Crônicas por Inquérito Telefônico (Vigitel), a prevalência de HAS no país chegou a $24,7 \%$ em $2018^{3}$. Vale ressaltar que na região metropolitana do município sede deste estudo (Maringá, Paiçandu e Sarandi) a prevalência de HAS, identificada em inquérito domiciliar com 1.232 pessoas adultas ( 18 a 59 anos), foi de $21,6 \% 4$.

Conforme aponta a literatura, vários fatores podem contribuir para a ocorrência da HAS, como aqueles considerados não modificáveis (herança genética, idade, sexo e etnia) e os modificáveis (ingestão de grande quantidade de sal, estresse, sedentarismo, uso de bebidas alcoólicas, tabagismo, entre outros) $)^{5}$. Os profissionais de saúde podem ajudar a prevenir a HAS e a manter a qualidade de vida da pessoa com a doença já instalada, mediante atuação focalizada nos fatores modificáveis ${ }^{5}$.

Estimativas apontam que aproximadamente $1 / 3$ das pessoas com HAS desconheça sua condição ${ }^{6}$. Além disso, em muitas situações, o diagnóstico ocorre somente no momento de uma complicação como infarto agudo do miocárdio ou acidente vascular encefálico, decorrente do descontrole da pressão arterial por vários anos ${ }^{6}$. Acrescenta-se a isso a baixa adesão ao tratamento ou sua realização de forma incorreta entre os que conhecem o diagnóstico, o que pode desencadear o surgimento de complicações e, por vezes, hospitalizações ou reospitalizações ${ }^{6}$.

Diante da magnitude dos problemas que envolvem essa doença, o Brasil, por meio do Ministério da Saúde, tem, há algumas décadas, desenvolvido várias ações e implantado políticas públicas e programas de cuidado à saúde, especialmente no contexto da Atenção Básica (AB), com o intuito de melhorar a efetividade da assistência prestada aos indivíduos com $\mathrm{HAS}^{7}$. Por exemplo, o Plano de Reorganização da Atenção à HAS e ao Diabetes Mellitus (DM), lançado em 2001, que fomentou o acesso e a vinculação dos usuários com hipertensão arterial aos serviços da $A B^{8}$. Ademais, com o objetivo de melhorar a qualidade da assistência à saúde no Sistema Único de Saúde (SUS), foram criados o Programa Nacional de Avaliação dos Serviços de Saúde, em 2004, e o Programa Nacional de Melhoria do Acesso e da Qualidade da Atenção Básica, em 2011, a fim de uniformizar o padrão de qualidade nas diferentes esferas de gestão9.

A partir de então, tomando-se como subsídio o resultado das avaliações da assistência, avanços ocorreram, sobretudo, nas normatizações acerca da rede de atenção à saúde e na operacionalização da regionalização. Apesar disso, ainda é preciso haver avaliações locorregionais com a finalidade de se traçar planos de ação para minimizar fragmentações ainda existentes e consolidação das linhas de cuidado em HAS ${ }^{10}$.

Em linhas gerais, os estudos com objetivo avaliativo investigaram as atividades desenvolvidas pelo Programa de Assistência ${ }^{11}$, a qualidade do acompanhamento de usuários com HAS ${ }^{12}$, o uso de diferentes serviços e o acesso a eles ${ }^{13}$, além da implementação das redes de atenção à saúde nessa população ${ }^{12}$. Contudo, ainda são escassos estudos que identifiquem e classifiquem o grau de implantação do Programa de Assistência à Pessoa com HAS, de modo global e sob os aspectos estruturais, da prática assistencial e da organização do serviço.

Neste sentido, considera-se necessário avaliar o modo como um programa está implantado nas dimensões envolvidas em sua operacionalização ${ }^{11}$ a fim de compreender seu funcionamento e os fatores que modificam sua dinâmica e, por consequência, a eficácia das ações desenvolvidas por esse programa. Diante disso, questiona-se: como se apresenta a 
implantação do Programa de Assistência às Pessoas com HAS nos municípios? Para responder ao referido questionamento, este estudo teve como objetivo avaliar o grau de implantação do Programa de Assistência às Pessoas com HAS no município de Maringá, Paraná.

\section{MÉTODO}

Pesquisa avaliativa e transversal, realizada no âmbito da $A B$ em Maringá, sede da $15^{\text {a }}$ Regional de Saúde, que engloba 30 municípios. Em 2013, a população de Maringá era de 367.410 habitantes. A rede de AB contava com 27 Unidades Básicas de Saúde (UBS), 65 equipes da Estratégia Saúde da Família (ESF), 1 equipe do Programa de Agente Comunitário de Saúde e 7 equipes dos Núcleos de Apoio à Saúde da Família.

A coleta de dados ocorreu entre abril e junho de 2013 por meio de entrevista estruturada com 63 enfermeiros da ESF, visto que 2 estavam afastados por causa de licença médica. A opção por abordar enfermeiros esteve relacionada ao fato de que, conforme mostra a literatura, esses profissionais exercem a função de coordenadores das equipes da ESF e normalmente participam do desenvolvimento das ações dos programas implementados na unidade, pois comumente apresentam menor rotatividade - atuação superior a cinco anos ${ }^{14}$.

As entrevistas foram previamente agendadas por telefone e realizadas em local reservado, nas próprias UBS. Durante sua realização, foi utilizado um instrumento construído especificamente para avaliar a implantação do Programa de Assistência às Pessoas com HAS ${ }^{15}$. $\mathrm{Na}$ construção do instrumento, que fez parte do estudo que originou o presente trabalho, foi tomado como referencial o modelo proposto por Hartz e Silva, constituído de sete etapas ${ }^{16}$. A definição dos critérios de análise incluídos no instrumento se deu com o uso da técnica Delphi, da qual participaram 12 especialistas (trabalhadores das equipes, gestores, especialistas em cardiologia e pesquisadores da área de avaliação), que atribuíram valor de 0 a 10 pontos a cada um dos critérios propostos na matriz de análise.

Só foram incluídos na versão final do instrumento os critérios que obtiveram média aritmética $\geq 7$, os quais foram considerados validados pelo consenso dos especialistas quanto à sua importância, sendo que, conforme maior a média aritmética, maior era a importância atribuída. O desvio-padrão serviu para estimar o grau ou a ausência do consenso. Assim, aqueles critérios que apresentavam desvio-padrão inferior a 3 e média maior ou igual a 7 foram considerados consensuais e inseridos na matriz de dimensões, como já estabelecido em outro estudo ${ }^{17}$.

O instrumento utilizado foi constituído de 121 questões e 3 dimensões: a estrutural, composta por 79 questões e 6 subdimensões (área física; materiais e equipamentos para consultas ambulatoriais; equipamentos de urgência; materiais para educação em saúde; insumos, exames e medicamentos; e recursos humanos); a prática assistencial, formada por 25 questões e 2 subdimensões (ações coletivas e atendimento individual); e a organização da atenção, constituída por 17 questões, sem subdimensões.

A tabulação dos dados ocorreu em planilha do Microsoft Office Excel', os quais, posteriormente, foram analisados no programa Statistica ${ }^{\circ}$ 6.09. Para a análise dos dados, foi calculada a pontuação ponderada de cada uma das três dimensões, atribuindo-se o mesmo peso para cada uma delas, tendo como base a média das notas atribuídas pelos especialistas. Como o número de questões em cada dimensão era diferente, o máximo de pontos que poderiam ser obtidos no conjunto (337 pontos) foi ponderado de tal forma que cada dimensão poderia alcançar um total de 112,33 pontos (1/3).

Cabe destacar que todas as questões das dimensões prática assistencial e organização da atenção valiam 3 pontos, e na dimensão estrutural 7 valiam 1 ponto, 16 valiam 2 pontos e as outras 56 valiam 3 pontos. Essa diferença decorreu das diferentes médias de pontuação atribuídas pelos especialistas convidados para a validação das questões, visto que as médias entre 7,0 e 7,9 correspondiam a 1 ponto; entre 8,0 e 8,9, a 2 pontos; e entre 9,0 e 10,0, a 3 pontos. Por fim, como o número de questões era diferente em cada dimensão, foi necessário definir o valor a ser atribuído às questões em cada dimensão. Por exemplo, os itens que valiam 3 pontos passaram a valer 0,78, 4,16 e 6,60 pontos, respectivamente, nas dimensões estrutural, prática assistencial e organização da atenção. 
O grau de implantação foi determinado a partir da média dos escores obtidos em termos percentuais em relação às três dimensões ${ }^{15}$. Dessa forma, a somatória dos pontos de todas as questões de uma mesma dimensão determinou o nível de implantação dela, o qual pode ser classificado em incipiente, intermediário e avançado. Assim, o primeiro tercil (até 33,33\%) representou o nível incipiente, o segundo tercil (33,34 a 66,66\%), o intermediário, e o terceiro (66,67 a 100\%), o nível avançado ${ }^{18}$.

A relação entre as três dimensões foi verificada por meio do coeficiente de correlação de Pearson e foi adotado 5\% como nível de significância. Para atender aos pressupostos do teste estatístico adotado, previamente a normalidade dos dados foi testada a partir do teste de Kolmogorov-Smirnov. Além disso, o pressuposto de homoscedasticidade também foi atendido.

Como foi constatada a normalidade dos dados referentes às dimensões avaliadas, foi feita a opção pela realização do teste paramétrico citado e pelo uso da média como medida de posição central que melhor representaria os dados. No entanto, para os dados referentes ao grau de implantação, conforme as 27 UBS avaliadas, foi adotado o uso das medianas e dos respectivos intervalos interquartílicos, visto que cada UBS apresentava um número pequeno de unidades observacionais $(n<50)$, representado pelas equipes de ESF, e, com isso, foi assumido que os dados não seguiam distribuição normal.

O estudo foi aprovado pelo Comitê Permanente de Ética em Pesquisas com Seres Humanos da instituição signatária, sob o Parecer no 168.220 . Todos os enfermeiros participantes assinaram, em duas vias, o Termo de Consentimento Livre e Esclarecido.

\section{RESULTADOS}

Dos 63 enfermeiros entrevistados, apenas 4 (6,34\%) eram do sexo masculino. 0 tempo de trabalho na ESF variou de 9 meses a 13 anos, com média de 6,3 anos. A maioria (82,5\%) trabalhava na mesma equipe da ESF há mais de três anos. Isso legitima a escolha deles como informantes do estudo. $\mathrm{O}$ grau de implantação do Programa de Assistência às Pessoas com HAS em Maringá foi considerado avançado apenas em 4 (6,3\%) das 63 equipes em estudo e intermediário nas demais. O escore médio obtido foi de 186,6 pontos (mínimo 146,3 e máximo $255,4)$ de 337 pontos possíveis. A dimensão estrutural foi aquela que obteve melhor avaliação, ao passo que a prática assistencial, a pior (Tabela 1).

A dimensão estrutural foi considerada avançada na totalidade das equipes, apesar do baixo desempenho das subdimensões "material para educação em saúde" e "área física", que sabidamente são indispensáveis no desenvolvimento das demais dimensões, mas compensadas pelas outras subdimensões classificadas como avançadas. Na subdimensão "área física", foi observada insuficiência em relação a itens como consultório para acolhimento e para consulta de enfermagem. Entre os "materiais e equipamentos", foram insuficientes esfigmomanômetros infantis e para usuários obesos. Já na subdimensão "materiais para educação em saúde", foi verificada ausência ou insuficiência de materiais didáticos para a implementação das atividades.

Em relação à dimensão prática assistencial, as equipes apresentaram situação bastante heterogênea, sendo que $68,3 \%$ delas foram classificadas como intermediárias, $25,3 \%$, como incipientes, e apenas 6,3\%, como avançadas. A subdimensão "ações coletivas" apresentou melhor desempenho que "atendimento individual". Isso porque os grupos de HIPERDIA eram realizados com frequência pelas equipes. Entre as "ações coletivas", foi observada baixa frequência de identificação de indivíduos com hipertensão em grupos de risco. E nos "atendimentos individuais", foram insuficientes a utilização de escalas de risco cardiovascular e a sistematização de planos de cuidado. Por fim, na dimensão organização da atenção, a maioria das equipes $(92,8 \%)$ apresentou grau de implantação intermediário.

A Figura 1 mostra que equipes de uma mesma unidade de saúde apresentam diferentes graus de implantação, caracterizado por um desvio-padrão elevado, o que denota a adoção de diferentes processos de trabalho na mesma UBS. Isso foi observado, principalmente, em duas unidades, as de número 7 e 25 . Em ambas, a estrutura física se mostrou adequada, e a diferença entre as equipes ocorreu nas dimensões prática assistencial e organização da atenção. 
Tabela 1. Classificação geral e por dimensão do grau de implantação do Programa de Assistência às Pessoas com Hipertensão Arterial Sistêmica nas equipes da Estratégia Saúde da Família. Maringá, Paraná, Brasil, 2013

\begin{tabular}{|c|c|c|c|c|c|c|}
\hline \multirow{3}{*}{ Dimensões } & \multicolumn{6}{|c|}{ Classificação $(n=63)$} \\
\hline & \multicolumn{2}{|c|}{ Incipiente } & \multicolumn{2}{|c|}{ Intermediário } & \multicolumn{2}{|c|}{ Avançado } \\
\hline & $\mathbf{n}$ & $\%$ & $\mathbf{n}$ & $\%$ & $\mathbf{n}$ & $\%$ \\
\hline $\begin{array}{l}\text { Classificação geral das } \\
\text { equipes }\end{array}$ & - & - & 59 & 93,6 & 04 & 6,3 \\
\hline Dimensão estrutural & - & - & - & - & 63 & 100,0 \\
\hline Área física & - & - & 28 & 44,4 & 35 & 55,5 \\
\hline Materiais e equipamentos & - & - & - & - & 63 & 100,0 \\
\hline Equipamentos para urgência & - & - & - & - & 63 & 100,0 \\
\hline $\begin{array}{l}\text { Materiais para educação em } \\
\text { saúde }\end{array}$ & 13 & 20,7 & 28 & 44,4 & 22 & 34,9 \\
\hline Recursos humanos & - & - & - & - & 63 & 100,0 \\
\hline $\begin{array}{l}\text { Dimensão práticas } \\
\text { assistenciais }\end{array}$ & 16 & 25,3 & 43 & 68,2 & 04 & 6,3 \\
\hline Promoção da saúde & 05 & 7,9 & 48 & 76,1 & 10 & 15,8 \\
\hline Atendimento individual & 16 & 25,3 & 43 & 68,2 & 04 & 6,3 \\
\hline $\begin{array}{l}\text { Dimensão organização da } \\
\text { atenção }\end{array}$ & 04 & 6,3 & 58 & 92,8 & 01 & 1,58 \\
\hline
\end{tabular}

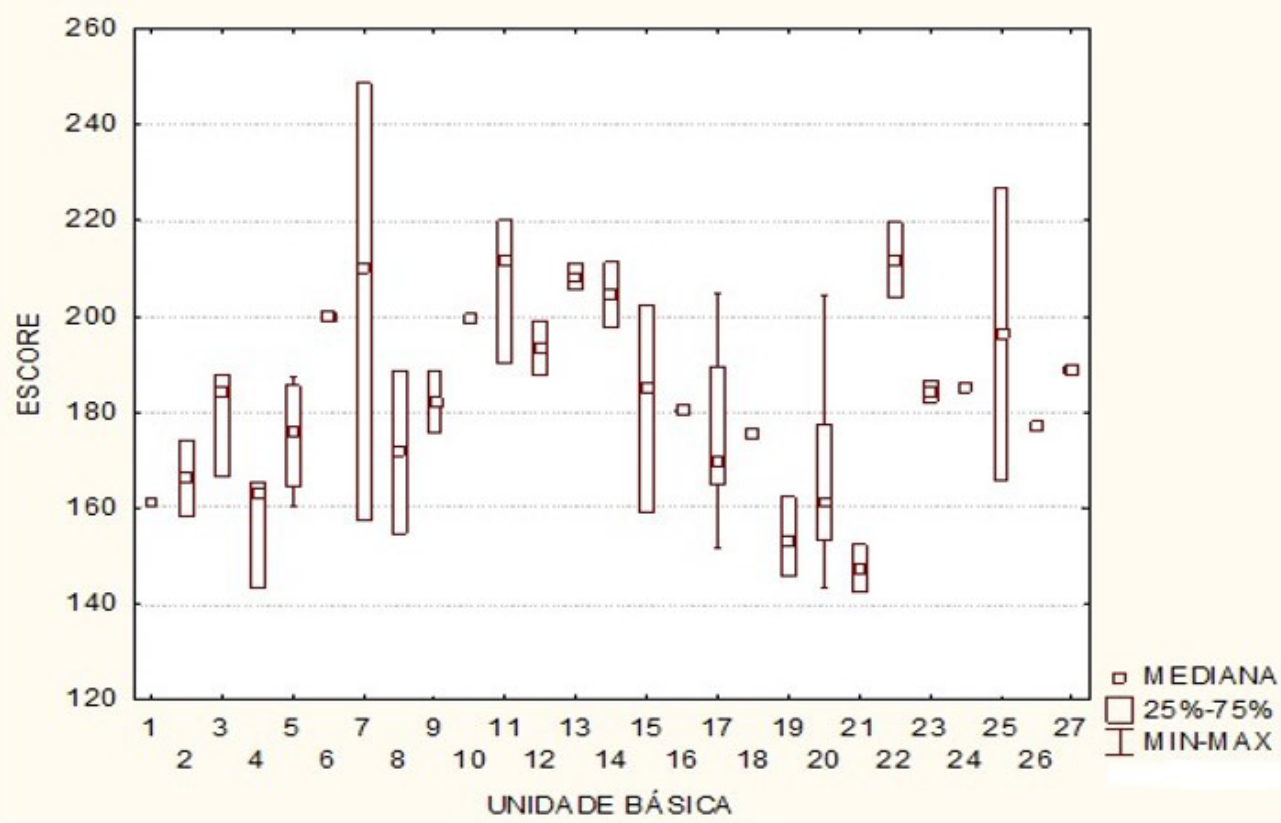

Figura 1. Escore mediano do grau de implantação do Programa de Assistência às Pessoas com Hipertensão Arterial Sistêmica por Unidade Básica de Saúde. Maringá, Paraná, Brasil, 2013.

Na análise de correlação de Pearson, entre as três dimensões (Tabela 2), foi observada existência de correlação positiva/direta entre as dimensões estrutural e prática assistencial e também entre prática assistencial e organização da atenção. Considerando que maiores 
Tabela 2. Análise da correlação entre as dimensões estrutura, prática assistencial e organização da atenção no Programa de Assistência às Pessoas com Hipertensão Arterial Sistêmica. Maringá, Paraná, Brasil, 2013

\begin{tabular}{lccc} 
& \multicolumn{2}{c}{ Correlação de Pearson } \\
\cline { 2 - 4 } Dimensões & Estrutural & Prática assistencial & $\begin{array}{c}\text { Organização da } \\
\text { atenção }\end{array}$ \\
\cline { 2 - 4 } Estrutural & 1,000 & 0,340 & 0,156 \\
\cline { 1 - 3 } p-valor & & 0,006 & 0,220 \\
\hline Prática assistencial & 0,340 & 1,000 & 0,300 \\
\hline p-valor & 0,006 & & 0,017 \\
\hline $\begin{array}{l}\text { Organização da } \\
\text { atenção }\end{array}$ & 0,156 & 0,300 & 1,000 \\
\hline p-valor & 0,220 & 0,017 & \\
\hline
\end{tabular}

pontuações obtidas nas dimensões representam melhores graus de implantação do Programa, destaca-se que as correlações positivas/diretas observadas indicam que, quanto melhor a implantação do Programa na dimensão estrutural, melhor foi a implantação na prática assistencial, e o mesmo se deu em relação a essa dimensão e organização da atenção.

\section{DISCUSSÃO}

Os dados mostraram que, na perspectiva dos enfermeiros, a maioria das equipes da ESF apresentou grau intermediário de implantação do Programa de Assistência às Pessoas com HAS. Ainda, foi verificado que existem fragilidades, principalmente quando se analisa a variabilidade dos escores obtidos, com base no grau de implantação por dimensão, visto que algumas dimensões e subdimensões avaliadas apresentaram maior frequência dos graus de implantação incipiente e intermediário.

Apesar disso, a dimensão estrutural foi classificada como avançada na totalidade das UBS, mostrando que, de modo geral, estas apresentam condições suficientes para a implantação do Programa, o que, entretanto, não significa necessariamente que todas estejam adequadas. Isso constitui indicativo positivo dos investimentos financeiros nessa área, principalmente com relação a recursos humanos, materiais e equipamentos, incluindo aqueles utilizados em situações de urgência.

Embora todas as equipes tenham sido consideradas avançadas, no que tange à estrutura quase metade delas foi classificada como intermediária em relação à "área física" e mais da metade (65\%) foi classificada como incipiente ou intermediária em relação à disponibilidade de "material para educação em saúde". Essas subdimensões estruturais podem implicar, consideravelmente, a prática assistencial e a organização da atenção, visto que estão diretamente relacionadas ao desenvolvimento de intervenções educativas individuais e coletivas que objetivem promover a saúde e prevenir complicações e agravos em pessoas com HAS ${ }^{19}$.

Em estudo realizado com enfermeiros da ESF, atuantes em município do Centro-Oeste brasileiro, foi constatado que déficits na estrutura física e falta de insumos e materiais educativos comprometiam a realização de atividades com as pessoas com HAS, pela restrição ao espaço físico das unidades e pela centralização das ações no profissional médico ${ }^{20}$. A indisponibilidade desses recursos, portanto, pode comprometer as ações de prevenção secundária (contra complicações da HAS), a promoção da saúde, o apoio ao autocuidado e a manutenção da adesão ao tratamento.

Assim, apesar de o município ter assumido desde 1994 a ESF como modelo assistencial e, recentemente, ter reformado ou construído grande parte de suas UBS, a área física, embora adequada, ainda apresenta deficiências para um atendimento centrado na figura do médico. 
Deficiências na estrutura física também foram identificadas como limitantes para a atenção às pessoas com HAS em Curitiba, Paraná, no que tange, propriamente, à disponibilidade de espaço ${ }^{12}$. Essa situação se torna relevante quando são observados que variados fatores, tais como a estrutura física e as possibilidades de acesso, podem influenciar a prática de ações educativas em pessoas com hipertensão arterial, requerendo de equipes e usuários a necessidade de buscarem meios efetivos para se alcançar a adesão ao tratamento nesse contexto ${ }^{21}$.

Em relação ao grau de implantação do Programa por UBS, foi verificado que equipes de uma mesma unidade apresentam diferentes graus de implantação. Logo, as equipes de saúde, em uma mesma unidade, adotam diferentes processos de trabalho. Esse fato revela que na organização da atenção, mesmo com estrutura comum, protocolos e procedimentos padrões definidos, os profissionais das equipes de saúde mantêm certa independência na execução do trabalho em saúde ou até mesmo vivenciam distintas realidades e dificuldades na efetivação da assistência.

De fato, cotidianamente, os trabalhadores tomam decisões que podem estar além ou aquém daquilo que se encontra normatizado para a qualidade da assistência prestada e, assim, alterar determinada etapa do processo de trabalho ou todo o processo. Desse modo, em uma mesma unidade de saúde atuam equipes que realizam a assistência de forma distinta, reafirmando a dinâmica e a complexidade do processo e gestão do trabalho em saúde. Deveras, para desenvolver uma assistência resolutiva com a população, as equipes devem planejar e coordenar as atividades, organizar os processos de trabalho e avaliar os resultados, sendo que, neste contexto, a estrutura pouco interfere na tomada de decisões ${ }^{22}$.

Com relação à dimensão práticas assistenciais, o número de equipes classificadas com grau de implantação incipiente merece atenção. Isso porque, apesar de o desenvolvimento de atividades de promoção à saúde ser parte das pactuações das esferas intergovernamentais, sobretudo com ênfase na mudança de comportamento da população brasileira - aumento da prática de atividade física de modo regular, estímulo à alimentação saudável e combate ao tabagismo -, apenas $15,8 \%$ das equipes se encontravam no grau avançado de implantação. Logo, aponta-se para um modelo assistencial no qual ainda não foram incorporados os princípios da saúde integral, com ênfase nas ações promocionais e assistenciais de saúde. É inconteste que o adequado controle tensional não pode se restringir unicamente à abordagem biopatológica do corpo doente. Pelo contrário, é mister considerar a historicidade e a subjetividade das pessoas no processo de adoecer cronicamente e de autocuidado ${ }^{17}$.

Como medida para suprir a necessidade de atenção no âmbito da assistência, ações voltadas à educação permanente das equipes podem constituir alternativas eficazes, pois a dinâmica e o objeto de trabalho na $A B$ apresentam constantes transformações, de modo que as equipes precisam consolidar e reorganizar saberes que coincidem com as necessidades observadas nesse âmbito de atenção em saúde ${ }^{23}$. Deste modo, acredita-se que gestores necessitam organizar em suas agendas gerenciais a pactuação de atividades formativas com setores comunitários públicos e/ou privados, a fim de favorecer a formação continuada dos profissionais.

Considera-se imperativa a necessidade de se discutir nessas atividades formativas a readequação das práticas assistenciais às pessoas com $H A S$ no contexto da $A B$, de acordo com o modelo de atenção às condições crônicas, calcada na prática do acolhimento extensivo, na estratificação do risco cardiovascular e na gestão da clínica, mediante a implantação do autocuidado apoiado e da gestão de caso, conforme a individualidade e a complexidade de cada caso ${ }^{24}$. Ademais, ações terapêuticas e de educação em saúde, desenvolvidas por meio de grupos de usuários, quando acrescidas às atividades individuais, estimulam a comunicação, a troca de experiências e a relação social, permitindo, assim, o apoio mútuo?.

A subdimensão "atendimento individual" obteve pior avaliação. É provável que isso seja decorrente do não seguimento adequado das diretrizes e normatizações contidas nos protocolos de assistência às pessoas com HAS, especialmente no que tange ao estabelecimento de prioridades na atenção e na periodicidade do acompanhamento com base na classificação de risco atribuída aos pacientes. Esses procedimentos são considerados imprescindíveis para 
determinar o plano terapêutico e dar a este o caráter individualizado necessário para qualificar a assistência. É a partir dessa classificação que são determinados os procedimentos a serem implementados pela equipe, garantindo, inclusive, a valorização da longitudinalidade do cuidado às doenças crônicas ${ }^{25}$.

Há que se destacar que a assistência às pessoas com HAS no município estudado se limitava à organização de grupos a cada três meses a fim de verificar a pressão arterial e o peso dos pacientes, bem como fornecer os medicamentos para o tratamento anti-hipertensivo. Atendimentos individuais, sobretudo com o médico, só ocorriam quando agendados por demanda espontânea, ou seja, apenas em casos de queixas pontuais e específicas, de modo que o estabelecimento e o acompanhamento de planos terapêuticos singulares ficavam prejudicados. Entretanto, ressalta-se ser importante dispensar maior atenção para qualificar a assistência às pessoas com HAS, de modo que os usuários do serviço adquiram maiores conhecimentos sobre sua doença e se sintam mais satisfeitos com a assistência recebida e, como consequência, sejam observados melhores resultados terapêuticos ${ }^{26}$.

No que se refere à dimensão organização da atenção, foi constatado que ela apresentou o menor número de equipes classificadas como avançadas. Apesar de o município ter realizado a adesão ao Programa HIPERDIA em 2002, apenas 17 equipes utilizavam informações provenientes dele para o cadastro e o monitoramento dos pacientes, bem como para o planejamento e a avaliação do Programa de Assistência às Pessoas com HAS. Esses resultados são semelhantes aos encontrados em Recife, em que o uso da informação para a organização e o planejamento de atividades assistenciais e burocráticas não constituía parte da rotina da maioria das equipes da ESF estudadas ${ }^{27}$.

Embora não tenha constituído foco do estudo, destaca-se que a integralidade dos dados lançados no sistema de informação é indispensável para um planejamento coerente com a realidade. No entanto, estudo realizado no Nordeste brasileiro constatou que a incompletude de dados obrigatórios do cadastro de pessoas com HAS no HIPERDIA foi elevada, e isso limitava a utilização dessas informações para o planejamento da assistência na $A B^{28}$. Esses resultados são importantes e preocupantes, pois mostram que o sistema de informações do Programa (SISHIPERDIA) era considerado pela maioria das equipes apenas como um procedimento burocrático de cadastramento das pessoas com HAS, e as informações, quando registradas, não subsidiavam o acompanhamento sistemático dessa parcela populacional. Assim, seu emprego como ferramenta de gestão e acompanhamento dos pacientes ainda é pouco usual.

No que se refere a uma avaliação ampliada, foi identificada correlação entre as dimensões estrutural e prática assistencial, o que coaduna, em parte, com os resultados de estudo realizado nesse mesmo município na década de 1990, que focalizou o processo de municipalização e a avaliação da qualidade das unidades de saúde. No referido estudo, foi constatado que a estrutura poderia até favorecer a assistência, mas tinha menos importância na definição da qualidade do serviço do que os componentes do processo e dos resultados ${ }^{29}$. Portanto, o presente estudo avança no sentido de apontar que a estrutura pode sim influenciar a atenção à saúde mais do que se supunha. Desta forma, deve-se considerar que a adequada manutenção da estrutura das unidades de saúde tem influência na prestação da assistência. E isso deve ser considerado pelos gestores em saúde.

A correlação identificada entre as dimensões práticas assistenciais e organização da atenção revelou que, quanto melhor as práticas assistenciais, melhor era a organização da assistência. Justifica-se tal correlação pelo fato de os itens que compunham cada dimensão convergirem para a assistência com qualidade às pessoas com HAS, isto é, itens da prática assistencial, como a periodicidade da realização de estratégias de promoção da saúde com vistas a identificar pessoas com HAS de acordo com fatores de risco, consultas segundo protocolo, estratificação do risco cardiovascular, visita domiciliar a usuários não aderentes e o estabelecimento de planos de cuidado sistematizados, podiam estar atrelados aos itens constituintes da organização da atenção, como a utilização do HIPERDIA para planejamento e avaliação do Programa, o sistema de referência e contrarreferência, o controle e a busca ativa de usuários faltosos e a periodicidade de agendamento de consultas médicas conforme a estratificação do risco cardiovascular. 
Tal correlação deve fazer parte do cotidiano do Programa de Assistência às Pessoas com HAS, visto que são elementos constantes na Linha Guia de Assistência à Hipertensão Arterial, elaborada pela Secretaria de Estado de Saúde do Paraná, que tem como objetivo balizar a mudança de abordagem às pessoas com HAS no estado, orientando, inclusive, os encaminhamentos entre $\mathrm{a} A \mathrm{~B}$ e a atenção especializada ${ }^{30}$.

Aponta-se que os resultados têm implicações diretas na prática assistencial e na gestão dos serviços de $A B$. Há a necessidade de os profissionais serem treinados e atuarem com base nas políticas e protocolos estabelecidos nacionalmente, mas também adaptados a cada realidade, no sentido de obter melhoria da qualidade e maior assertividade na assistência às pessoas com HAS. Considera-se que as distintas dimensões que alicerçam o Programa devem estar em graus de implantação equiparados, de modo que a atenção possa ser dispensada de forma constante e equânime.

É importante destacar que a presente investigação possui limitações. A primeira delas decorreu do fato de não terem sido incorporados gestores, demais profissionais das equipes e usuários. A segunda se refere à não avaliação da dimensão "resultados" do Programa de Assistência às Pessoas com HAS. Esses dois aspectos impediram uma avaliação multiprofissional e mais abrangente. Assim, os resultados obtidos estão condicionados às percepções que os enfermeiros possuíam acerca das dimensões de estrutura e processo. Contudo, os achados favorecem a reflexão sobre as fragilidades relacionadas à implantação do Programa no município, as quais certamente interferem em seu funcionamento.

Por fim, sugere-se que novos estudos avaliativos, em distintas partes do Brasil, sejam realizados com a incorporação de trabalhadores de outras categorias profissionais, gestores e usuários, além de agregar no instrumento uma dimensão relativa aos resultados alcançados com a implantação do Programa. Essa triangulação de informantes e o aumento de variáveis investigadas têm potencial para ampliar os resultados da presente pesquisa e fortalecer o conhecimento sobre o processo de implantação de programas voltados à saúde das pessoas com HAS. Isso, por sua vez, pode propiciar a melhora na assistência prestada a essa parcela populacional, ao se identificarem os gargalos que dificultam a implantação e o funcionamento dos programas.

\section{REFERÊNCIAS}

1. Talaei M, Sadeghi M, Mohammadifard N, Shokouh P, Oveisgharan S, Sarrafzadegan N. Incident hypertension and its predictors: the Isfahan Cohort Study. J Hypertens. 2014;32(1):30-8. http://dx.doi.org/10.1097/ HJH.0b013e32836591d4. PMid:24048009.

2. Malta DC, Gonçalves RPF, Machado IE, Freitas MIF, Azeredo C, Szwarcwald CL. Prevalência da hipertensão arterial segundo diferentes critérios diagnósticos, Pesquisa Nacional de Saúde. Rev Bras Epidemiol. 2018;21(Supl. 1):e180021. http://dx.doi.org/10.1590/1980-549720180021.supl.1. PMid:30517472.

3. Brasil. Ministério da Saúde. Vigilância de fatores de risco e proteção para doenças crônicas por inquérito telefônico - Vigitel Brasil 2018. Brasília; 2019.

4. Arruda GO, Santos AL, Teston EF, Cecílio HPM, Radovanovic CAT, Marcon SS. Association between selfreported health and sociodemographic characteristics with cardiovascular diseases in adults. Rev Esc Enferm USP. 2015;49(1):61-8. http://dx.doi.org/10.1590/S0080-623420150000100008. PMid:25789643.

5. Malta DC, Bernal RTI, Andrade SSCA, Silva MMA, Velasquez-Melendez G. Prevalência e fatores associados com hipertensão arterial autorreferida em adultos brasileiros. Rev Saude Publica. 2017;51(Supl. 1):11s. PMid:28591346.

6. Solbiati VP, Oliveira NRC, Teixeira CVLAS, Gomes RJ. Adesão ao tratamento para prevenir agravos relacionados à hipertensão arterial e ao diabetes. Rev. Bras Obes Nutr Emagr. 2018;12(73):629-33.

7. Barreto MS, Silva RDT, Waidman MAP, Marcon SS. A trajetória das políticas públicas de saúde para Hipertensão Arterial Sistêmica no Brasil. Revista de APS. 2013;16(4):460-8.

8. Rêgo AS, Radovanovic CAT. Adesão/vínculo de pessoas com hipertensão arterial na Estratégia Saúde da Família. Rev Bras Enferm. 2018;71(3):1030-7. PMid:29924169.

9. Salci MA, Paiano M, Radovanovic CAT, Carreira L, Meirelles BHS, Silva DMVG. O programa de avaliação da atenção básica na ótica de profissionais de saúde e gestores. Rev Rene. 2019;20:e33980. http://dx.doi. org/10.15253/2175-6783.20192033980. 
10. Santos CM, Barbieri AR, Gonçalves CCM, Tsuha DH. Avaliação da rede de atenção ao portador de hipertensão arterial: estudo de uma região de saúde. Cad Saude Publica. 2017;33(5):e00052816. http:// dx.doi.org/10.1590/0102-311x00052816. PMid:28614449.

11. Radigonda B, Souza RKT, Cordoni L Jr, Silva AMR. Avaliação do acompanhamento de pacientes adultos com hipertensão arterial e ou diabetes melito pela Estratégia Saúde da Família e identificação de fatores associados, Cambé-PR, 2012. Epidemiol Serv Saude. 2016;25(1):115-26. http://dx.doi.org/10.5123/S167949742016000100012. PMid:27861684.

12. Silva JVM, Mantovani MF, Kalinke LP, Ulbrich EM. Avaliação do Programa de Hipertensão Arterial e Diabetes Mellitus na visão dos usuários. Rev Bras Enferm. 2015;68(4):626-32. http://dx.doi.org/10.1590/00347167.2015680408i. PMid:26422045.

13. Tanaka OY, Drumond M Jr, Gontijo TL, Louvison MCP, Rosa TEC. Hipertensão arterial como condição traçadora para avaliação do acesso na atenção à saúde. Cien Saude Colet. 2019;24(3):963-72. http:// dx.doi.org/10.1590/1413-81232018243.07312017. PMid:30892517.

14. Rocha BS, Munari DB, Bezerra ALQ, Melo LKA. Enfermeiros coordenadores de equipe do Programa Saúde da Família: perfil profissional. Rev Enferm UERJ. 2009;17(2):229-33.

15. Silva RLDT. Avaliação da implantação da assistência às pessoas com hipertensão arterial em município do Estado do Paraná [tese]. Maringá: Programa de Pós-graduação em Enfermagem, Universidade Estadual de Maringá; 2013. 149 p.

16. Hartz ZMA, Silva LMV. Avaliação em saúde: dos modelos teórico à prática na avaliação de programas e sistemas de saúde. Rio de Janeiro: FIOCRUZ; 2005.102 p

17. Souza LEPF, Silva LMV, Hartz ZMA. Conferência de consenso sobre a imagem-objetivo da descentralização da atenção à saúde no Brasil. In: Hartz ZMA, Silva LMV, editors. Avaliação em saúde: dos modelos teóricos à prática na avaliação de programas e sistemas de saúde. Rio de Janeiro: FIOCRUZ; 2005. p. 86-102.

18. Silva LMV, Esperidião MA, Viana SV, Alves VS, Lemos DVS, Caputo MC, et al. Avaliação da implantação de programa voltado para melhoria da acessibilidade e humanização do acolhimento aos usuários na rede básica: Salvador, 2005-2008. Rev Bras Saúde Mater Infant. 2010;10(1 Supl. 1):s131-43. http://dx.doi. org/10.1590/S1519-38292010000500012.

19. Manoel MF, Marcon SS, Baldissera VDA. Estratégias educativas para pessoas com hipertensão arterial e diabetes mellitus. Rev Enferm UERJ. 2013;21(3):403-8.

20. Pedrosa ICF, Corrêa ACP, Mandú ENT. Influências da infraestrutura de centros de saúde nas práticas profissionais: percepções de enfermeiros. Cienc Cuid Saúde. 2011;10(1):58-65. http://dx.doi.org/10.4025/ cienccuidsaude.v10i1.13288.

21. Machado JC, Cotta RMM, Moreira TR, Silva LS. Análise de três estratégias de educação em saúde para portadores de hipertensão arterial. Cien Saude Colet. 2016;21(2):611-20. http://dx.doi.org/10.1590/141381232015212.20112014. PMid:26910168.

22. Dias RM, Moniz MA. Competências gerenciais do enfermeiro na estratégia saúde da família: percepção de graduandos de enfermagem. Rev Pesq: Cuidado é Fundamental Onl. 2019;11(4):1048-52.

23. Ramos WT, Quiulo LD, Andrade LDF. A educação permanente no âmbito da atenção primária à saúde: uma revisão integrativa. Braz J Hea Rev. 2018;1(1):35-45.

24. Mendes EV. O cuidado das condições crônicas na atenção primária à saúde: o imperativo da consolidação da estratégia saúde da família. Brasília: Organização Pan-Americana da Saúde; 2012. 512 p.

25. Mendez RDR, Santos MAD, Wysocki AD, Ribeiro BDB, Stauffer LF, Duarte SJH. Estratificação do risco cardiovascular entre hipertensos: influência de fatores de risco. Rev Bras Enferm. 2018;71(4):1985-91. http://dx.doi.org/10.1590/0034-7167-2017-0528. PMid:30156687.

26. Barreto MS, Reiners AAO, Marcon SS. Knowledge about hypertension and factors asso-ciated with the nonadherence to drug therapy. Rev Lat Am Enfermagem. 2014;22(3):491-8. http://dx.doi.org/10.1590/01041169.3447.2442.

27. Costa JMBS, Silva MRF, Carvalho EF. Avaliação da implantação da atenção à hipertensão arterial pelas equipes de saúde da família do município do Recife (PE, Brasil). Cien Saude Colet. 2011;16(2):623-33. http://dx.doi.org/10.1590/S1413-81232011000200026. PMid:21340338.

28. Correia LOS, Padilha BM, Vasconcelos SML. Completude dos dados de cadastro de portadores de hipertensão arterial e diabetes mellitus registrados no Sistema Hiperdia em um estado do Nordeste do Brasil. Cien Saude Colet. 2014;19(6):1685-97. http://dx.doi.org/10.1590/1413-81232014196.02842013. PMid:24897470.

29. Scochi MJ. Avaliando o cuidado ao hipertenso em serviços de saúde. Acta Sci Health Sci. 2001;23(3):739-44.

30. Paraná. Secretaria do Estado de Saúde. Superintendência de Atenção à Saúde. Linha guia de hipertensão arterial. Curitiba: SESA; 2014. 